\section{Possible Mechanisms for Delayed Migration of the Closed Cell-Designed Enterprise Stent When Used in the Adjunctive Treatment of a Basilar Artery Aneurysm}

With great interest, we read the 2 articles documenting delayed migration of the self-expanding intracranial microstent-the Enterprise (Cordis, Miami Lakes, Florida). ${ }^{1,2}$ Meanwhile, Lavine et $\mathrm{al}^{3}$ had recorded another delayed migration of the Enterprise stent their article in Neurosurgery. These events of delayed migration all occurred with the Enterprise stent, whereas they never happened with the Neuroform stents (Boston Scientific, Natick, Massachusetts) on the basis of our experience and the experience of Kelly et al. ${ }^{1}$ Two events took place after the aneurysm had been coiled, whereas the third delayed migration occurred for a staged coiling procedure after the stent had been deployed. What are the possible mechanisms for the delayed migration?

First, the structural characteristics of the Enterprise stent play a dominant role. Both the Neuroform and the Enterprise stents are soft self-expanding microstents especially designed for intracranial use in the adjunctive treatment of wide-neck aneurysms. The major difference between these 2 stents is the structural design. The Neuroform stent is of open cell or half open cell design, while the Enterprise stent is of closed cell design with flared ends to enhance apposition to the wall. Each end of the Enterprise stent has 4 radiopaque markers that flare out for fixation of the stent onto the vascular wall when fully deployed. Its closed cell design makes it work as a whole body; thus, a force used at one end will be transmitted to the other end immediately. For a stent with open cell or semi-open cell design like the Neuroform stent, each segment can serve as a separate fixing device, like the flared ends of the Enterprise stent, to enhance apposition of the stent to the wall, and a force used at one end will not be transmitted to the other end so easily. This probably explains why there is no delayed migration of the Neuroform stent.

Second, the diameter difference between the posterior cerebral artery (PCA) and the basilar artery (BA) produces a squeezing force in the artery with the smaller diameter-the PCA. Because of this smaller diameter, the distal end of the stent cannot be fully flared to enhance fixation, and a force is produced to squeeze the stent proximally.

Third, the angle formed between the PCA and the BA will be expanded after stent placement. We have noticed the phenomenon of angle expansion in our daily practice in the use of not only the Enterprise stent but also the Neuroform stent (Fig 1). In addition, the vascular angles were measured before and after stent placement and at follow-up of the patients reported to have delayed migration of the Enterprise stent (Fig 2). ${ }^{1-3}$ The angles formed between the stented PCA and BA all expanded after stent placement. In the report by Rodriguez et al, ${ }^{2}$ no prestenting figure was given, but angular reduction after the stent migrated completely into the BA compared with the angle at the end of the stent-coiling procedure demonstrated that stent placement could cause the angle to expand. What significance does the expansion of stented angle have? After angular expansion, the stented segment of the PCA will become closer to a line with the $\mathrm{BA}$, thus decreasing resistance of stent migration proximally.

Fourth, some part of the deployed stent was located in the BA, which is in a perpendicular position with the ground, and the weight of this part of stent will drag the stent downward. Fifth, the movement of the head may also play a role in the delayed proximal migration of the Enterprise stent.

Besides those mentioned above, another phenomenon may also help explain why delayed stent migration never takes place with the Neuroform stent. Benndorf et $\mathrm{al}^{4}$ reported an increased cell opening and prolapse of struts of a Neuroform stent in curved vasculature after a study with angiographic CT. A Neuroform stent was deployed for the treatment of a BA aneurysm in a 46-year-old woman. After the stent was deployed from the BA to the right PCA, a CT scan demonstrated increased cells and prolapse of struts at the aneurysm site. Moreover, the open cell-designed Neuroform stent and the closed cell Enterprise stent perform differently when deployed in a curvature.

Ebrahimi et $\mathrm{al}^{5}$ studied stent conformity in curved vascular mod-
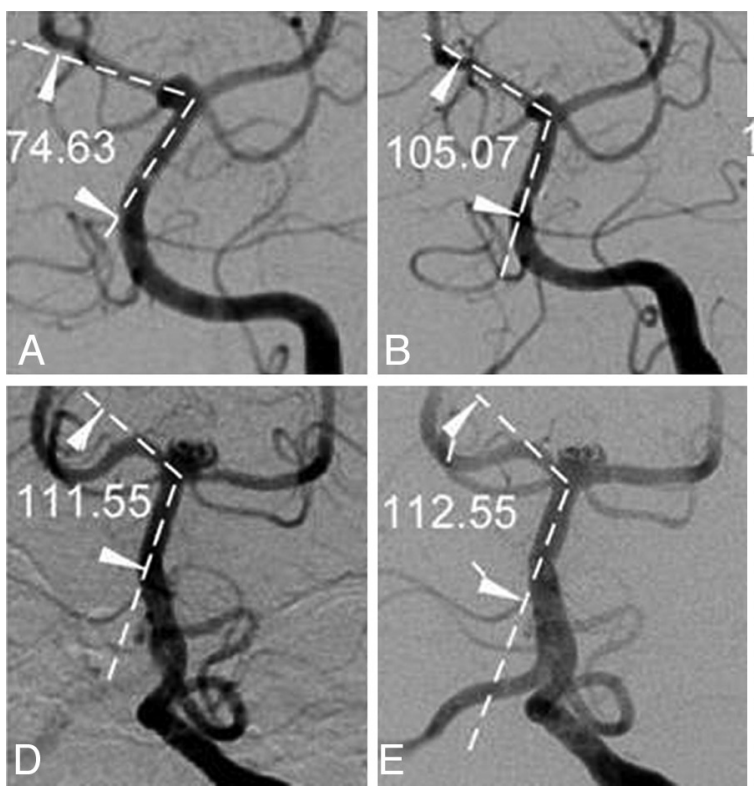
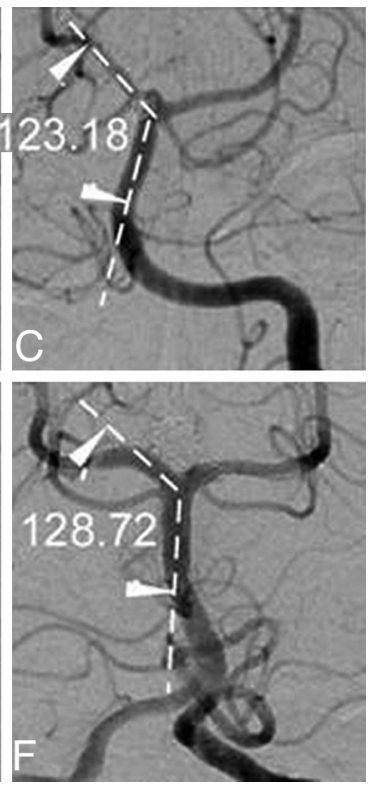

Fig 1. Stent placement caused angles to expand. $A-C, A$ 62-year-old female patient had an Enterprise stent deployment for the treatment of a small BA aneurysm. The angle between the right PCA and the BA was $74.63^{\circ}$ before stent placement $(A), 105.07^{\circ}$ immediately following stent placement $(B)$, and $123.18^{\circ} 7$ months later. $D-F$, 377 -year-old female patient had 2 Neuroform stents deployed in a Y-configuration to treat a BA aneurysm. The angle between the right PCA and the BA was $111.55^{\circ}$ before stent placement, $112.55^{\circ}$ immediately after stent placement, and $128.72^{\circ}$ at 4 -year follow-up. 


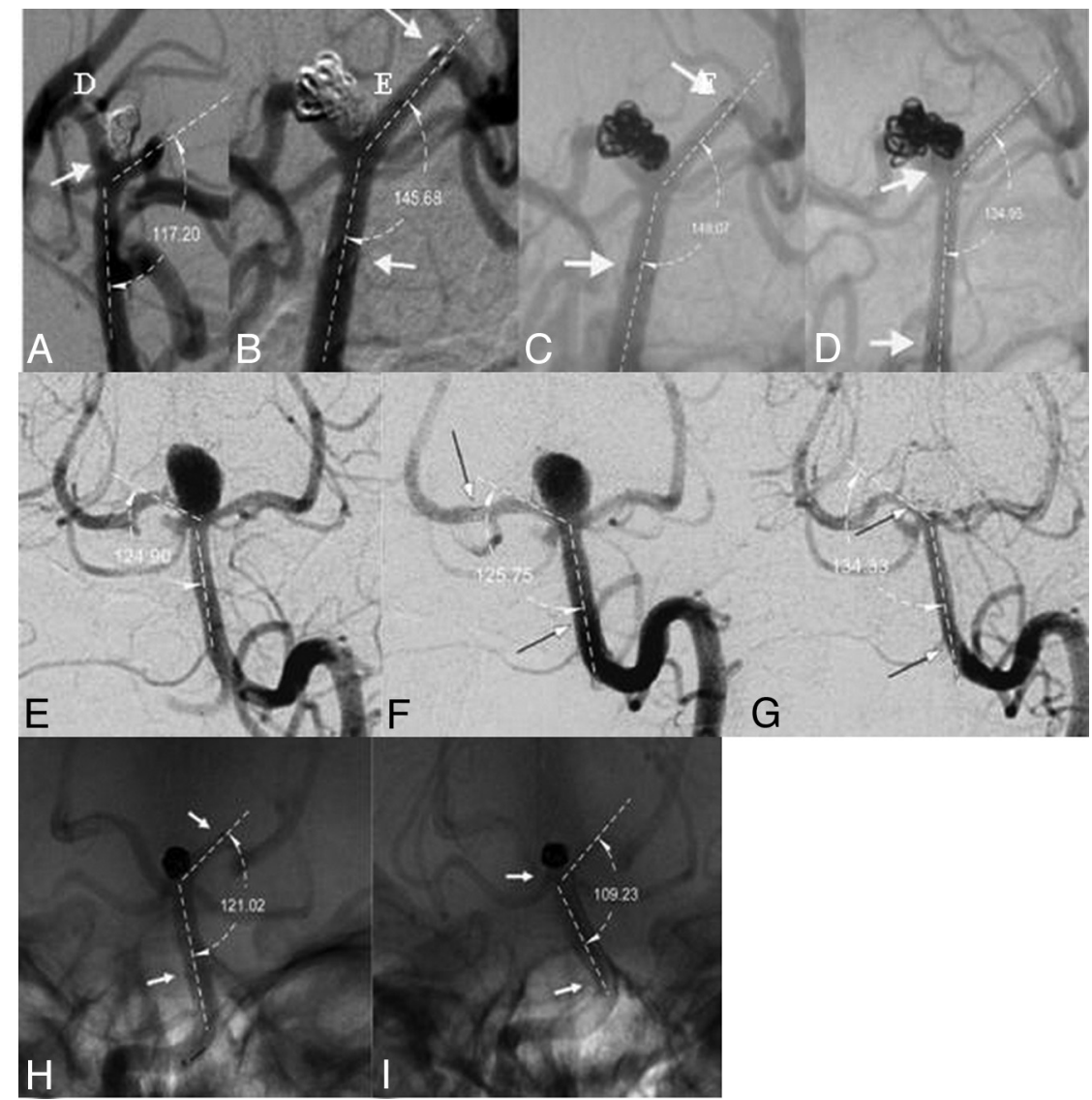

Fig 2. Modified figures show angular changes before and after stent placement and after delayed stent migration. $A-D$, The angle between the left PCA and the $B A$ was $117.2^{\circ}$ before stent placement $(A), 145.68^{\circ}$ at the end of stent-coiling procedure $(B), 148.07^{\circ}$ at 3-month follow-up $(C)$, and $134.95^{\circ}$ after delayed stent migration at 5-month follow-up $(D)$. Modified with permission from Kelly et al. ${ }^{1} E-G$, The angle between the right PCA and the BA was $124.90^{\circ}$ before stent placement $(E), 125.75^{\circ}$ after stent placement $(F)$, and $134.33^{\circ}$ after delayed proximal stent migration $(G)$. Modified with permission from Lavine et al. ${ }^{3} \mathrm{H}-\mathrm{l}$, The angle between the left PCA and the BA was $121.02^{\circ}$ after stent placement $(H)$ and $109.23^{\circ}$ after the stent migrated into the BA 6 months later ( $)$. Modified with permission from Rodriguez et al. ${ }^{2}$

els with simulated aneurysm necks by using flat panel CT, with the following results gained: The Neuroform 2 Treo stent showed a continuous increase in cell size, an outward prolapse at the convexity, and an inward prolapse of struts at the concavity of the curvature, whereas the Enterprise stent showed an increasing trend to flatten and to kink with curvatures that are more acute. These observations demonstrate that the Enterprise stent will maintain a constant shape or opening as a whole in a curvature while the Neuroform stent tends to kink. The kinking phenomenon of the Neuroform stent shows that each segment of this stent acts separately, not as a whole, and that a force used at one end will not be transmitted to the other end in a quick and immediate way. Although the kinking phenomenon of an open cell stent may contribute to stent thrombosis; in-stent stenosis; herniation of coils into the parent artery; and entanglement with coils, guidewires, microcatheters, or other devices, subsequently resulting in possible complications, this phenomenon helps prevent delayed stent migration.

Although a closed cell stent may become flattened at its midsection at a curved vessel, a kinking phenomenon of the stent will occur only at $\leq 30^{\circ}$ of curvature of the vessel according to a report. ${ }^{5}$ Most curved cerebral vessels are not at an acute angle, which suggests that the said kinking phenomenon will probably not occur when a closed cell-designed stent is applied in the cerebral vasculature for the treatment of intracranial aneurysms.

In our daily practice in treating aneurysms at the basilar tip, the Neuroform stent is usually used. Sometimes, the Enterprise stent may be used together with the Neuroform stent in the Y-configuration. When used this way, the 2 stents will entangle to prevent proximal migration of any single stent. However, a good understanding of the performance of any stent after deployment will ensure the freedom from possible complications, including delayed stent migration.

\section{References}

1. Kelly ME, Turner RD, Moskowitz SI, et al. Delayed migration of a self-expanding intracranial microstent. AJNR Am J Neuroradiol 2008;29:1959-60

2. Rodriguez GJ, Maud A, Taylor RA. Another delayed migration of an Enterprise stent. AJNR Am J Neuroradiol 2009;30:E57

3. Lavine SD, Meyers PM, Connolly ES, et al. Spontaneous delayed proximal migration of Enterprise stent after staged treatment of wide-necked basilar aneurysm: technical case report. Neurosurgery 2009;64:E1012, discussion E1012

4. Benndorf G, Claus B, Strother CM, et al. Increased cell opening and prolapse of struts of a Neuroform stent in curved vasculature: value of angiographic computed tomography-technical case report. Neurosurgery 2006;58:ONS-E380, discussion ONS-E380

5. Ebrahimi N, Claus B, Lee CY, et al. Stent conformity in curved vascular models with simulated aneurysm necks using flat panel CT: an in vitro study. AJNR Am J Neuroradiol 2007;28:823-29

B. Gao

A.M. Malek

Cerebrovascular and Endovascular Division Department of Neurosurgery Tufts University Medical Center Boston, Massachusetts

DOI 10.3174/ajnr.A2258 